\title{
Defining the Characteristics of Chronic Hypoparathyroidism Not Adequately Controlled on Conventional Therapy: Consensus Findings of Three European Delphi Panels
}

\author{
Kamran Iqbal · Nin Dass · Christina Gip · Juan Vila · Angela J. Rylands • \\ Claudio Marelli
}

Received: May 30, 2019 / Published online: October 5, 2019

(C) The Author(s) 2019

\section{ABSTRACT}

Introduction: European Society of Endocrinology (ESE) guidelines provide goals for hypoparathyroidism management but do not define characteristics of chronic hypoparathyroidism that is not adequately controlled. Three European country-specific Delphi panels were conducted to gain consensus on these characteristics.

Enhanced Digital Features To view enhanced digital features for this article go to https://doi.org/10.6084/ m9.figshare.9741620.

Electronic supplementary material The online version of this article (https://doi.org/10.1007/s12325019-01102-5) contains supplementary material, which is available to authorized users.

K. Iqbal · N. Dass

Shire Pharmaceuticals Ltd, a member of the Takeda group of companies, London, UK

C. Gip

Shire Sweden AB, a member of the Takeda group of companies, Stockholm, Sweden

J. Vila

Shire Pharmaceutical Iberia, a member of the Takeda group of companies, Madrid, Spain

A. J. Rylands

OPEN VIE, Marlow, UK

C. Marelli $(\bowtie)$

Shire International $\mathrm{GmbH}$, a member of the Takeda group of companies, Zug, Switzerland

e-mail: Claudio.marelli@takeda.com
Methods: Delphi panels were conducted in the UK, Sweden, and Portugal using similar methodology. At each round, panellists considered patients with chronic hypoparathyroidism whose disease is not adequately controlled on conventional therapy according to a matrix of four presentations of patients with chronic hypoparathyroidism: normal biochemical levels/well (group 1), abnormal biochemical levels/well (group 2), normal biochemical levels/unwell (group 3), and abnormal biochemical levels/unwell (group 4), with wellness defined by the patient's persistent symptoms, comorbidities, and complications. For groups 2-4, panellists rated characteristics in five categories (patient characteristics, family history, comorbidities, biochemistry, and symptoms/impact on quality of life [QoL]) with respect to defining a patient as having chronic hypoparathyroidism that was not adequately controlled on conventional therapy. Consensus was achieved when more than $80 \%$ of respondents agreed.

Results: Among the three countries, panellists agreed that characteristics within four of the five categories (patient characteristics, comorbidities, biochemistry, and symptoms/impact on QoL) were important for defining inadequate control. Characteristics deemed important in groups 2-4 included a history of compliance problems and chronic kidney disease stages 4 and 5. In groups 2 and 4, the biochemical parameters deemed important were 
serum calcium, urinary calcium, and serum creatinine. In groups 3 and 4 , tingling or numbness in the hands/feet and face was the only symptom deemed important in all three countries.

Conclusion: Delphi panels conducted in three European countries provided national consensus on key parameters of patient characteristics, biochemistry, comorbidities, and symptoms/ impact on QoL that define not adequately controlled chronic hypoparathyroidism. These characteristics should be tested more widely for their applicability in clinical practice.

Funding: Shire International $\mathrm{GmbH}, \mathrm{Zug}$, Switzerland, a member of the Takeda group of companies.

Keywords: Chronic hypoparathyroidism treatment; Consensus; Delphi panels; Endocrinology; ESE guidelines

\section{INTRODUCTION}

Chronic hypoparathyroidism is a rare endocrine disorder characterised by absent or inappropriately low levels of endogenous parathyroid hormone, resulting in impaired mineral homeostasis [1]. Many patients with hypoparathyroidism are managed with conventional therapy of orally administered calcium and active vitamin D [2-4]; however, some remain in a disease state that is not adequately controlled [2, 4, 5]. Despite conventional treatment, a subset of patients continues to experience symptoms of fatigue, muscle cramps, paraesthesia, and complications (e.g. nephrocalcinosis, chronic renal disease) that negatively affect quality of life (QoL) $[1,3,6,7]$.

Recent guidelines from the European Society of Endocrinology (ESE) [3] and the First International Conference on the Diagnosis, Management and Treatment of Hypoparathyroidism [4] outline recommendations for the diagnosis, treatment, and monitoring of chronic hypoparathyroidism in adults. The guidelines include general goals for the management of hypoparathyroidism but do not provide a standardised definition for inadequate control in patients treated with conventional therapy.
Management guidelines are largely centred on maintaining appropriate biochemical levels, including serum calcium; 24-h urinary calcium; and serum phosphate, magnesium, and vitamin D. In addition, the patients who require high doses of orally administered calcium and/or vitamin D (e.g. $>2.5 \mathrm{~g}$ of calcium and/or $>1.5 \mu \mathrm{g}$ of 1,25 -dihydroxyvitamin $\mathrm{D}_{3}$ or $>3.0 \mu \mathrm{g}$ alfacalcidol) to maintain serum calcium within an appropriate range are considered to have disease that is not adequately controlled [4]. Although biochemical levels and supplement dosing are important, the guidelines also emphasise the importance of recognising the symptoms, complications, and impact on QoL associated with chronic hypoparathyroidism. However, there is a lack of a European consensus regarding which symptoms and complications are relevant in defining patients whose disease is not adequately controlled. In addition, regional differences in patient management exist across healthcare systems. The objective of these three European countryspecific Delphi panels was to obtain national consensus regarding the characteristics that define patients with chronic hypoparathyroidism whose disease is not adequately controlled on conventional therapy.

\section{METHODS}

\section{Patient Presentation Matrix}

Fourteen endocrinologists, endocrine surgeons, and nephrologists convened to provide a framework to characterise the severity and inadequate control of disease in clinical practice. These experts proposed a patient presentation matrix to define the severity of chronic hypoparathyroidism. The matrix uses two axes, biochemical levels and wellness (includes symptoms, comorbidities, and complications), to define four groupings of adult patients with hypoparathyroidism. This study did not take into account any effect of comedications related to hypoparathyroidism.

Abnormal biochemistry was defined as meeting any of the following criteria: albumenadjusted serum calcium levels $<2.0 \mathrm{mmol} / \mathrm{L}$ on 
at least 3-4 annual measurements despite $\geq 3 \mu \mathrm{g}$ /day of active vitamin $\mathrm{D}$; broad fluctuations in serum calcium concentrations without apparent cause but with considerable time outside the normal range; serum phosphate concentrations $>1.6 \mathrm{mmol} / \mathrm{L}$; and 24-h urinary calcium excretion $>7.5 \mathrm{mmol} / 24 \mathrm{~h}$ in men and $>6.25 \mathrm{mmol} / 24 \mathrm{~h}$ in women.

Patient wellness was defined on the basis of the severity of hypoparathyroidism-related symptoms, comorbidities, and complications. Severity was classified into three categories: disease-specific symptoms and signs (those independently indicative of severe hypoparathyroidism; e.g. seizures, hypocalcaemic hospitalisations, progressing nephrocalcinosis, chronic kidney disease [CKD] stages 4 and $5,>2.5 \mathrm{~g}$ orally administered calcium daily); disease-related symptoms and signs (those indicative of severe hypoparathyroidism if at least two appear together, occur repeatedly, and substantially impair QoL; e.g. paraesthesia, cramps, brain fog/memory loss, nonprogressing nephrocalcinosis, nonprogressing impaired estimated glomerular filtration rate); and general, nonspecific symptoms and signs (those indicative of severe hypoparathyroidism according to the physician's discretion and taking the whole history of the patient into account [e.g. cardiac arrhythmias, anxiety, fatigue] and nonspecific comorbidities [e.g. obesity, hypertension, atherosclerosis, acute cardiovascular events, depression, malabsorption syndromes]).

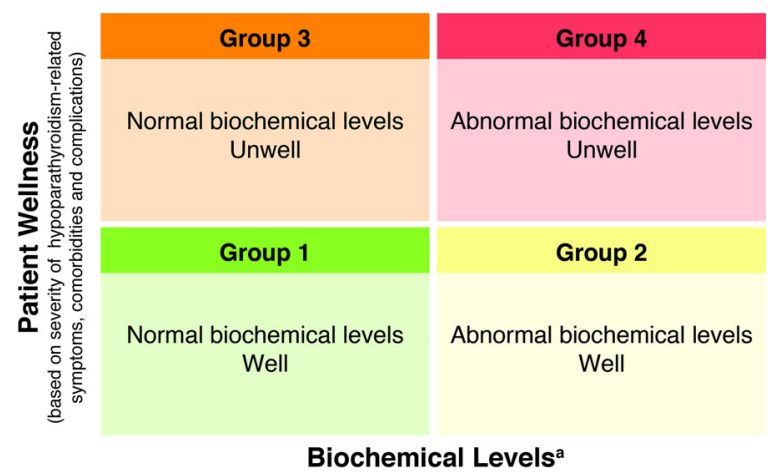

Fig. 1 Patient presentation matrix. ${ }^{a}$ Biochemical levels viewed independently of patients' symptomatology
According to the proposed patient presentation matrix (Fig. 1), the following four patient groupings were identified: group 1, patients with normal biochemistry who feel well; group 2 , patients with abnormal biochemistry who feel well; group 3, patients with normal biochemistry who feel unwell; and group 4, patients with abnormal biochemistry who feel unwell.

\section{Study Design}

The Delphi panel method is a group facilitation technique designed to achieve agreement by group consensus $[8,9]$. The Delphi technique aims to attain confluence of opinion from knowledge-area experts using iterative but anonymous voting to assess group opinion. Overall and individual results from each round of voting are reviewed by Delphi panellists before the initiation of subsequent voting rounds.

Delphi panels were employed in the UK, Sweden, and Portugal to determine the characteristics of patients with chronic hypoparathyroidism that is not adequately controlled on conventional therapy, according to the patient presentation matrix. In all three countries, consensus via the Delphi method was sought through a series of structured rounds, conducted either as a face-to-face meeting or as an electronic self-administered questionnaire. In the UK, a three-round process was used comprising a face-to-face meeting followed by two rounds of an electronic, self-administered questionnaire. In Sweden, a modified tworound process was used in which the electronic questionnaire was administered first, followed by a face-to-face discussion in which areas of agreement and disagreement were explored (no rating of characteristics occurred). After the panel discussion, a second questionnaire was administered. A two-round process was used in Portugal consisting of a face-to-face meeting, followed by an electronic self-administered questionnaire. At each Delphi round, consensus was defined as at least $80 \%$ of respondents in agreement. All three Delphi panels were conducted sequentially and independently, and 
were blinded to each other. The study did not involve any interventions with human participants and are therefore exempt from institutional review board approval. All panellists consented to participate in the Delphi panels and complete the survey. However, as all data were anonymised and aggregated, and as individual responses could not be identified or attributed to the panellists, consent for publication was not required.

\section{Participants}

A total of 20 participants with expertise in hypoparathyroidism were invited to serve on the Delphi panels. Panellists were invited by vendors facilitating the Delphi panels (UK: OPEN VIE, an OPEN Health company, Marlow; Portugal: Exigo Consultores, Lisbon; Sweden: PAREXEL International, Stockholm). Because hypoparathyroidism is a rare disease with a small number of medical experts, Shire, a member of the Takeda group of companies, provided vendors with a list of hospitals with known expertise in treating hypoparathyroidism or names of physicians with demonstrated experience managing hypoparathyroidism, a publication history related to hypoparathyroidism, or current participation in advocacy groups for patients with hypoparathyroidism. In the UK, Shire directly approached all experts on hypoparathyroidism who were then invited by vendors. All participants received a modest financial incentive for participation offered by the study sponsor. Shire was blinded to panellist responses. In the UK, 10 endocrinologists from across the country participated. In Sweden, five clinical endocrinologists active at university hospitals throughout Sweden were recruited. In Portugal, participants included four endocrinologists and one endocrine surgeon.

\section{Questionnaire and Panel Interaction}

The first panel was conducted in the UK. The questionnaire was developed by identifying variables associated with chronic hypoparathyroidism based on the published literature and later validated in interviews with clinical experts; patient perspective was captured by taking key descriptors from the patient support and advocacy website Hypopara UK (www. hypopara.org.uk). To ensure that the terminology included in the questionnaire was relevant to patients, for the first round in the UK a patient expert was engaged face-to-face to provide input. The UK questionnaire was then used in Sweden and Portugal following validation from a local country perspective. A copy of the full questionnaire used in Sweden is provided as an example (Table $S 1$ in the supplementary material).

In all three countries, participants were asked to consider the level of importance of various characteristics for defining chronic hypoparathyroidism that is not adequately controlled on conventional therapy for groups 2-4 in the patient presentation matrix. In the UK, characteristics were rated on a five-point Likert scale during round one, a three-point Likert scale during round two, and a binary response during round three. In Sweden, characteristics were rated on a five-point Likert scale during round one and a three-point Likert scale during round two. In Portugal, characteristics were rated on a five-point Likert scale during round one. In round two, characteristics for which consensus was obtained in round one were rated on a binary response scale ("agree" or "disagree"); characteristics for which consensus was not obtained in round one were rated on a three-point Likert scale. For several questions, the electronic self-administered questionnaires also included an open field for participants to expand on their responses. Characteristics were grouped into the following categories: patient characteristics, family history, comorbidities, biochemistry, and patient-reported symptoms/ impact on QoL.

\section{Data Analysis}

Descriptive statistics were used and tabulation of data is presented in this manuscript. 


\section{RESULTS}

\section{Characteristics of Patients with Chronic Hypoparathyroidism Not Adequately Controlled with Conventional Therapy}

The Delphi panellists' estimated proportion of patients with chronic hypoparathyroidism across the patient presentation matrix is shown in Table 1 . The characteristics that achieved consensus as "very important" or "important" when considering a patient whose disease is not adequately controlled on conventional therapy are shown in Table S2 (supplementary material). None of the characteristics deemed "very important" were common within the three countries for groups $2-4$ of the patient presentation matrix. However, in group 4 (patients with abnormal biochemistry who felt unwell), serum calcium was found to be "very important" in the UK and Sweden, and gastrointestinal disorder associated with malabsorption was "very important" in Sweden and Portugal.

Commonalities in characteristics deemed "very important" or "important" in all three countries for groups $2-4$ of the patient presentation matrix are shown in Fig. 2. In group 2 (patients with abnormal biochemistry who felt well), commonalities were found in the patient characteristics, comorbidities, and biochemistry categories; no symptom characteristics were deemed "important" because the wellness status of these patients was considered normal. In group 3 (patients with normal biochemistry who felt unwell), one commonality each was found in the categories of patient characteristics (history of compliance problems) and patientreported symptoms/impact on QoL (tingling or numbness in the hands or feet and in the face); no biochemical characteristics were deemed "important" because the biochemical status of these patients was considered normal. In group 4 (patients with abnormal biochemistry who felt unwell), commonalities were found in the patient characteristics, comorbidities, biochemistry, and patient-reported symptoms/impact on QoL categories. In groups 2 and 4, four specific renal comorbidities (stone risk, CKD stages 4 and 5, renal calcifications, and hypercalciuria) were identified as "very important" or "important," whereas in group 3 no renal comorbidities were identified in all three countries. Although family history of endocrine disorders was deemed an "important" characteristic in groups 2-4 within Sweden, this was not the case in the other two countries.

\section{DISCUSSION}

To our knowledge, this is the first publication reporting the results of Delphi panels

Table 1 Delphi panellists' estimated proportion of patients with chronic hypoparathyroidism across the patient presentation matrix

\begin{tabular}{llll}
\hline Patient presentation matrix & \multicolumn{2}{l}{ Estimated proportion of patients, \% } \\
\cline { 2 - 4 } & UK & Sweden & Portugal \\
\hline Group 1 & 62 & 59 & 68 \\
Group 2 & 13 & 10 & 15 \\
Group 3 & 14 & 15 & 8 \\
Group 4 & 12 & 7 & 9 \\
Unknown & - & 9 & - \\
\hline
\end{tabular}

Panellists provided the level of importance of characteristics for defining chronic hypoparathyroidism that is not adequately controlled on conventional therapy for groups $2-4$; however, the patient presentation matrix is a tool to assist physicians in the definition of "not adequately controlled" and it does not infer that all patients in groups $2-4$ are not adequately controlled as these are only estimates provided by the Delphi panellists 


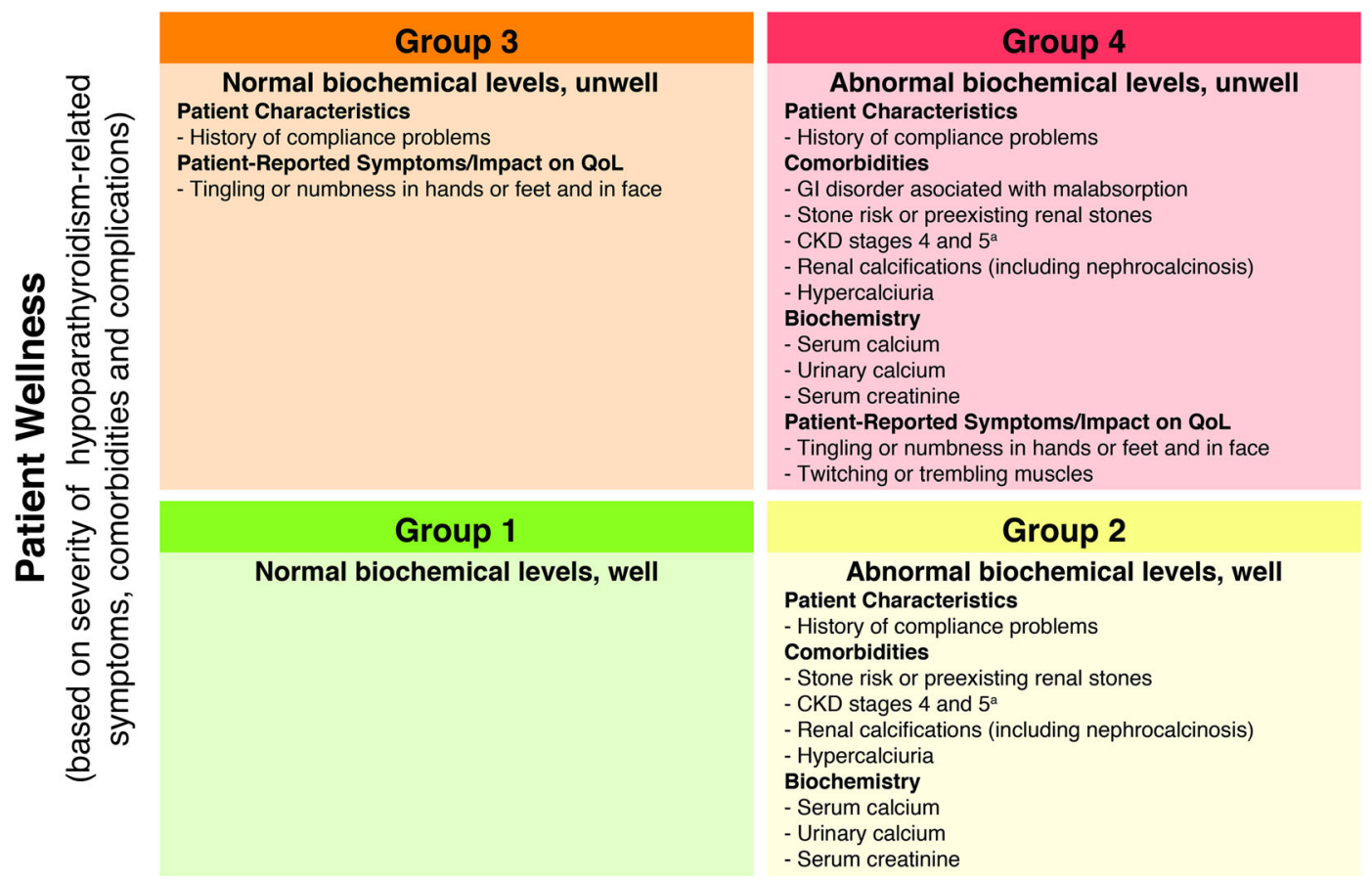

Biochemical Levels

Fig. 2 Characteristics that achieved consensus as "very important" or "important" in all three countries for groups 2-4 of the patient presentation matrix. CKD chronic

conducted independently, but sharing similar methodology, to obtain consensus regarding the characteristics that define patients with chronic hypoparathyroidism whose disease is not adequately controlled on conventional therapy. Despite the lack of a consensus definition for not adequately controlled chronic hypoparathyroidism, in clinical practice it is often assessed on the basis of biochemistry, specifically maintenance of serum calcium within a target range $[3,4]$. However, mineral homeostasis extends beyond control of serum calcium [3], and clinical evaluation of patients with hypoparathyroidism must consider more than just biochemistry. Interestingly, Al-Sharefi et al. recently reported the applicability of a "no calcium" regimen in a cohort of 24 patients with hypoparathyroidism who had previously experienced adverse effects from calcium supplements [10]. However, there is a discernible difference between the two studies. The cohort of patients in Al-Sharefi et al. appears to be not well managed whereas the focus of this kidney disease, GFR glomerular filtration rate, GI gastrointestinal, $Q o L$ quality of life. ${ }^{\mathrm{a} D e f i n e d}$ as GFR $<30 \mathrm{~mL} / \mathrm{min} / 1.73 \mathrm{~m}^{2}$ on dialysis

manuscript is patients with chronic hypoparathyroidism that is not adequately controlled with conventional therapy. Nevertheless, these results corroborate previous findings that chronic hypoparathyroidism managed with conventional therapy (i.e. orally administered calcium and/or supplementation with active forms of vitamin D) may exacerbate the risk of hypercalciuria and ectopic soft tissue calcification, including nephrolithiasis and nephrocalcinosis [4, 11-13]. Defining disease control or severity in hypoparathyroidism is challenging, in part because it can refer to either the current status of the patient or the prognosis of the patient. In addition, disease severity is subjective, given that patients experience symptoms differently, and is multifactorial, in that comorbidities can substantially affect the severity of the condition.

In an effort to provide a clinically applicable means of defining chronic hypoparathyroidism that is not adequately controlled, a group of clinicians with expertise in hypoparathyroidism 
developed the patient presentation matrix (Fig. 1). This matrix of four quadrants combines the biochemistry, symptoms, comorbidities, complications, and medication burden dimensions of the disease to create a tool that is consistent with the general management goals of chronic hypoparathyroidism outlined in the European guidelines $[3,4]$ while also providing a framework that begins to differentiate severity within the patient population. Using the Delphi method, we sought characteristics of patients with chronic hypoparathyroidism that is not adequately controlled on conventional therapy for groups $2-4$ of the matrix.

Specific characteristics that were considered "very important" or "important" in defining patients who are not adequately controlled on conventional therapy varied within the three countries for groups 2-4, which may reflect country-specific considerations for chronic hypoparathyroidism. Nonetheless, characteristics that achieved consensus as "very important" or "important" included factors related to patient characteristics, comorbidities, biochemistry, and patient-reported symptoms/impact on QoL. The only patient characteristic common in all three countries in all three patient groups was history of compliance problems.

In groups 2 and 4, comorbidities pertaining to renal function were found to be "very important" or "important" characteristics in all three countries in defining chronic hypoparathyroidism that was not adequately controlled. The importance of renal comorbidities is consistent with the current guidelines for treatment goals of hypoparathyroidism $[3,4]$, which aim to avoid renal complications, including hypercalciuria, impaired renal function, nephrolithiasis, and calcifications.

In groups 2 and 4 , serum calcium, urinary calcium, and serum creatinine were found to be "very important" or "important" biochemical characteristics that helped define chronic hypoparathyroidism that was not adequately controlled in all three countries. No biochemical characteristics were deemed "important" in group 3 within all three countries because these patients had normal biochemical levels. Routine monitoring of serum calcium, urinary calcium, and creatinine is consistent with the current treatment guidelines [3, 4]. In groups 2 and 4, the importance of serum phosphate and calcium-phosphate product as characteristics that define chronic hypoparathyroidism that is not adequately controlled on conventional therapy varied by country [3]. Portugal was the only country in which both of these parameters were deemed "important" in groups 2 and 4 . In Sweden, serum phosphate was deemed "important" in group 2 and "very important" in group 4, whereas in the UK, neither parameter achieved consensus as "important" or "very important." Differences among the three countries with regard to the importance of phosphate and calcium-phosphate product in defining chronic hypoparathyroidism that was not adequately controlled may reflect traditional disease management with conventional therapy of orally administered calcium supplements and active vitamin D; conventional treatment focuses on serum calcium control but does not fully address overall mineral homeostasis, including that of phosphate, which is under the physiological regulation of endogenous parathyroid hormone [14, 15]. According to ESE guidelines [3], disease management strategies should focus on how to better achieve normal mineral homeostasis, beyond just maintaining serum calcium control. Although consensus within the three countries was not reached regarding the importance of serum phosphate, some patients might fail to meet biochemical targets defined by the ESE guidelines for this parameter [5], highlighting the need to monitor serum phosphate in clinical practice.

In groups 3 and 4 , tingling or numbness in the hands or feet and in the face was found to be a "very important" or "important" symptom characteristic that defines disease that is not adequately controlled in all three countries. In group 4, twitching or trembling muscles was also found to be "important". According to the ESE guidelines [3], patients with hypoparathyroidism should be educated regarding symptoms of paraesthesia and spasms/twitches because they are associated with hypocalcaemia. These findings highlight the importance of incorporating patient-reported 
symptomatology into disease characterisation and management.

Collectively, these findings stand to inform an area of clinical practice that is currently limited in information. The patient presentation matrix provides a clinically relevant framework to assess each individual case. In particular, the biochemical parameters on the $\mathrm{x}$-axis are measurable and their normal ranges are derived from the existing guidelines, and the wellness parameters on the y-axis can also be objectively assessed and measured. In this study, consensus was gained on identified characteristics for defining not adequately controlled chronic hypoparathyroidism, and these characteristics were aligned to the four quadrants in the patient presentation matrix. Overall, this approach provides a preliminary framework which can be further refined in realworld clinical settings; it could be used by clinicians for monitoring patients and to aid in treatment decision-making.

This analysis is not without limitations. First, the findings reported here may not be accurate for all patients, and consensus does not necessarily imply correctness. Although chronic hypoparathyroidism is a rare disease, the selection of participants was subjective and thus may lend itself to bias. Additionally, although the overall methodology was similar for all three countries, there were some minor differences in the design of the three panels. The use of an open field in some of the questionnaire responses may also have introduced bias by the study researcher during interpretation of the findings. Furthermore, Delphi panellists were asked to estimate the percentage of patients in each quadrant of the patient presentation matrix which does not constitute a formal epidemiological study. In addition, the patient presentation matrix is a tool to assist physicians in the definition of "not adequately controlled" and it does not infer that all patients in groups 2,3 , and 4 are not adequately controlled as these are only estimates provided by the Delphi panellists. A final limitation is that expert opinion, even consensus expert opinion, does not rank high in the hierarchy of clinical evidence $[16,17]$. Despite these caveats, the Delphi method is well established for accommodating the input of a range of experts across widespread geographic areas and generating consensus on complex multidisciplinary topics [9]. Specific to this study, good participation rates from national experts enabled us to demonstrate agreement on characteristics of not adequately controlled chronic hypoparathyroidism, addressing a knowledge gap in the published literature.

\section{CONCLUSION}

Delphi panels conducted in these three countries provide a national consensus around characteristics that define patients with chronic hypoparathyroidism that is not adequately controlled on conventional therapy with calcium and active vitamin D. General consensus was achieved among the experts within the three countries on key parameters of patient characteristics (groups 2-4: history of compliance problems), biochemistry (groups 2 and 4: serum calcium, urinary calcium, and serum creatinine), comorbidities (groups 2 and 4: CKD stages 4 and 5, stone risk, renal calcifications, hypercalciuria; group 4 only: gastrointestinal disorder associated with malabsorption), and patient-reported symptoms/impact on QoL (group 3 and 4: tingling or numbness in the hands or feet and in the face; group 4 only: twitching or trembling muscles). In particular, consensus was reached regarding the high importance of serum calcium level in determining chronic hypoparathyroidism that was not adequately controlled. The list of characteristics derived from the Delphi panels should be tested more widely for their applicability in clinical practice.

\section{ACKNOWLEDGEMENTS}

The authors wish to acknowledge the following endocrinologists, nephrologist, and endocrine surgeons who developed the patient presentation matrix: Jens Bollerslev (Norway), Maria L. Brandi (Italy), Henning Dralle (Germany), Erik Fink Eriksen (Norway), William D. Fraser (UK), Pascal Houillier (France), Michael Mannstadt 
(USA), Giovanna Mantovani (Italy), Simon H.S. Pearce (UK), Antonio Sitges-Serra (Spain), Heide Siggelkow (Germany), and Lars Rejnmark (Denmark). We thank the Delphi panellists for their participation. We also thank Hypopara UK for their contributions to the Delphi panel during the early stages of the survey development.

Funding. Sponsorship for this study, the Rapid Service, and Open Access Fees were provided by Shire International GmbH, Zug, Switzerland, a member of the Takeda group of companies.

Editorial Assistance. Editorial support, funded by Shire International GmbH Shire, a member of the Takeda group of companies, was provided by Maryann T. Travaglini, PharmD, of Complete Healthcare Communications, LLC, a CHC Group company (North Wales, PA, USA).

Authorship. All named authors meet the International Committee of Medical Journal Editors (ICMJE) criteria for authorship for this article, take responsibility for the integrity of the work as a whole, and have given their approval for this version to be published. All authors had full access to all of the data in this study and take complete responsibility for the integrity of the data and accuracy of the data analysis.

Prior Presentations. A portion of these data was presented previously at the 6th Swedish National Health Economics Conference, March 14-15, 2017, Linköping, Sweden, by M. Virhage, M. Hultberg, E. Medin, and C. Gip (not published) and at the Society for Endocrinology BES, November 6-8, 2017, Harrogate, UK by A. Rylands, K. Patel, N. Dass, and K. Iqbal (Endocrine Abstracts. 2017;50:abstr P062).

Disclosures. Kamran Iqbal is an employee of Shire, a member of the Takeda group of companies. Nin Dass is an employee of Shire, a member of the Takeda group of companies. Christina Gip is an employee of Shire, a member of the Takeda group of companies. Juan Vila is a former employee of Shire, a member of the
Takeda group of companies; his current affiliation is Sobi SL, Madrid, Spain. Angela J Rylands is an employee of OPEN VIE, an OPEN Health company, which was contracted to conduct this research in the UK by Shire, a member of the Takeda group of companies. Claudio Marelli is an employee of Shire, a member of the Takeda group of companies.

Compliance with Ethics Guidelines. The questionnaires did not involve any interventions with human participants and are therefore exempt from institutional review board approval. All panellists consented to participate in the Delphi panels and complete the survey. However, as all data were anonymised and aggregated, and as individual responses could not be identified or attributed to the panellists, consent for publication was not required.

Data Availability. The datasets generated and/or analysed during the current study are available from the corresponding author in deidentified form on reasonable request.

Open Access. This article is distributed under the terms of the Creative Commons Attribution-NonCommercial 4.0 International License (http://creativecommons.org/licenses/ by-nc/4.0/), which permits any noncommercial use, distribution, and reproduction in any medium, provided you give appropriate credit to the original author(s) and the source, provide a link to the Creative Commons license, and indicate if changes were made.

\section{REFERENCES}

1. Shoback D. Hypoparathyroidism. N Engl J Med. 2008;359:391-403.

2. Bilezikian JP, Brandi ML, Cusano NE, et al. Management of hypoparathyroidism: present and future. J Clin Endocrinol Metab. 2016;101:2313-24.

3. Bollerslev J, Rejnmark L, Marcocci C, et al. European Society of Endocrinology clinical guideline: treatment of chronic hypoparathyroidism in adults. Eur J Endocrinol. 2015;173:G1-120. 
4. Brandi ML, Bilezikian JP, Shoback D, et al. Management of hypoparathyroidism: summary statement and guidelines. J Clin Endocrinol Metab. 2016;101:2273-83.

5. Meola A, Vignali E, Matrone A, Cetani F, Marcocci C. Efficacy and safety of long-term management of patients with chronic post-surgical hypoparathyroidism. J Endocrinol Invest. 2018;41:1221-6.

6. Bilezikian JP, Khan A, Potts JT Jr, et al. Hypoparathyroidism in the adult: epidemiology, diagnosis, pathophysiology, target-organ involvement, treatment, and challenges for future research. J Bone Miner Res. 2011;26:2317-37.

7. Hadker N, Egan J, Sanders J, Lagast H, Clarke BL. Understanding the burden of illness associated with hypoparathyroidism reported among patients in the PARADOX study. Endocr Pract. 2014;20:671-9.

8. Hasson F, Keeney S, McKenna H. Research guidelines for the Delphi survey technique. J Adv Nurs. 2000;32:1008-15.

9. Hsu C-C, Sandford BA. The Delphi technique: making sense of consensus. Pract Assess Res Eval. 2007;12(10):1-8.

10. Al-Sharefi A, Glenister E, Morris M, Quinton R. Is calcium supplementation always needed in patients with hypoparathyroidism? Clin Endocrinol (Oxf). 2019;90:775-80.

11. Mitchell DM, Henao MP, Finkelstein JS, BurnettBowie SA. Prevalence and predictors of vitamin D deficiency in healthy adults. Endocr Pract. 2012;18:914-23.

12. Shoback DM, Bilezikian JP, Costa AG, et al. Presentation of hypoparathyroidism: etiologies and clinical features. J Clin Endocrinol Metab. 2016;101:2300-12.

13. Abate EG, Clarke BL. Review of hypoparathyroidism. Front Endocrinol (Lausanne). 2017;7:172.

14. Bergwitz C, Juppner $H$. Regulation of phosphate homeostasis by PTH, vitamin D, and FGF23. Annu Rev Med. 2010;61:91-104.

15. Jacquillet G, Unwin RJ. Physiological regulation of phosphate by vitamin $\mathrm{D}$, parathyroid hormone (PTH) and phosphate (Pi). Pflugers Arch. 2019;471:83-98.

16. Evans D. Hierarchy of evidence: a framework for ranking evidence evaluating healthcare interventions. J Clin Nurs. 2003;12:77-84.

17. Eddy DM. Evidence-based medicine: a unified approach. Health Aff (Millwood). 2005;24:9-17. 